\section{Macrophage Activation Syndrome Treated with Anakinra}

\section{To the Editor:}

A 32-year-old woman originally from Cambodia (living in Canada since 1993) developed symmetrical polyarthritis involving hands, wrists, and knees. Rheumatoid factor (RF) and anticyclic citrullinated peptide (CCP), antinuclear antibody (ANA), and anti-double-stranded DNA (dsDNA) were negative. A diagnosis of seronegative polyarthritis was made. Treatment was initiated with methotrexate, hydroxychloroquine, and sulfasalazine.

Three years later she developed tuberculosis of the spine (Pott's disease). She underwent surgical debridement and internal fixation of lumbar spine (T12-L1). Antituberculous drugs were initiated. She developed hepatitis secondary to isoniazid and was treated with ethambutol, pyrazinamide, and rifampin for a year, followed by pyrazinamide and rifampin maintenance therapy indefinitely.

Four years after the diagnosis of seronegative arthritis, she was readmitted to our hospital with fever $\left(39^{\circ} \mathrm{C}\right)$ and a flare of arthritis. She also presented a sterile pharyngitis, polyadenopathy, and small bilateral pleural effusions. No rash was noted during febrile episodes but she is naturally tanned. Laboratory results showed inflammatory anemia, neutrophilia, and elevated ferritin, sedimentation rate, and C-reactive protein (Table 1). Repeat RF, anti-CCP, and anti-dsDNA examinations were negative and ANA were weakly positive (1/320). Articular radiographs showed distal interphalangeal joint erosions and midcarpal erosions. Bone marrow biopsy was normal. A diagnosis of adult-onset Still's disease (AOSD) was made. Prednisone doses of $20 \mathrm{mg}$ BID were necessary to abate temperature. Because of her recent surgical instrumentation for vertebral tuberculosis, adding a biologic agent was feared. Weekly intramuscular gold salts were started ${ }^{1}$. She developed a rash and gold salts were stopped after 3 months. Prednisone was gradually tapered.

Two days after a prednisone taper to $5 \mathrm{mg}$ twice a day, she developed spiking fever $\left(39.5^{\circ} \mathrm{C}\right)$, increasing joint pain, headache, and diffuse myalgias. She was readmitted to hospital. Examination disclosed polyadenopathy, hepatosplenomegaly, and synovitis of all distal interphalangeal joints, most proximal interphalangeal joints and both wrists. She also had hypotension (blood pressure 80/40 $\mathrm{mm} \mathrm{Hg}$ ). Laboratory results (Table 1) disclosed pancytopenia and increased ferritin, mildly elevated liver enzymes (aspartate aminotransferase $61 \mathrm{U} / \mathrm{l})$, hypofibrinogenemia (1.47 $\mathrm{g} / \mathrm{l}$ ), normal coagulation measurements, and normal triglycerides. A bone marrow biopsy disclosed moderate to severe hemophagocytosis (Figure 1). A diagnosis of macrophage activation syndrome (MAS) was made. Anakinra $100 \mathrm{mg}$ subcutaneously daily was initiated, along with pulse methylprednisolone $1 \mathrm{~g} /$ day for 3 days, then prednisone $25 \mathrm{mg}$ twice daily. A dramatic response was observed. Within 48 hours of anakinra treatment, blood count increased and fever abated.

Two months after the diagnosis of MAS, the patient was feeling well with anakinra $100 \mathrm{mg}$ daily and prednisone $5 \mathrm{mg}$ twice daily. She had no more fever and only mild residual joint pain and no synovitis. Ten months after the initiation of anakinra, she is well and has minimal morning stiffness but no other symptoms. Her spinal disease is stable. She also remains on rifampin and pyrazinamide, as well as methotrexate $20 \mathrm{mg}$ subcutaneously once a week, folic acid, hydroxychloroquine $200 \mathrm{mg}$ daily, and prednisone $2 \mathrm{mg}$ per day on a slowly tapering regimen.

This case represents an atypical presentation of AOSD, with seronegative arthritis presenting 4 years before fever. At diagnosis, fever is present in $85 \%-100 \%$ of cases, and arthritis in $64 \%-100 \%{ }^{2}$. The rate of joint disease presenting without fever and evolving to AOSD is unknown. AOSD should be kept in mind in the differential diagnosis of seronegative arthritides.

MAS is a much feared complication of $\mathrm{AOSD}^{3}$. This syndrome is part of the histiocytic disorders, and represents an exaggerated activation of the reticuloendothelial system, in which histiocytes (tissue macrophages) phagocytize hematopoietic cells $s^{4,5}$. Mortality rate is $8 \%-22 \%{ }^{5}$. Various triggers have been reported, among them the use of antituberculous agents and gold salts ${ }^{5}$. These might have been triggers in our patient, but the use of antituberculous drugs was longstanding, and gold salts were stopped before the initiation of the syndrome. The prednisone taper with secondary increase of systemic inflammation might also have been responsible. The diagnosis of MAS is sometimes difficult. Diagnostic criteria have been described in children with systemic juvenile idiopathic arthritis ${ }^{6,7}$. Our case fulfilled these criteria, but there are no diagnostic criteria for adults. It is probable that AOSD and MAS represent a continuum of disease with varying degrees of severity, and that MAS is underdiagnosed in $\mathrm{AOSD}^{4,8}$.

Table 1. Maximal daily temperature, laboratory values, and treatments.

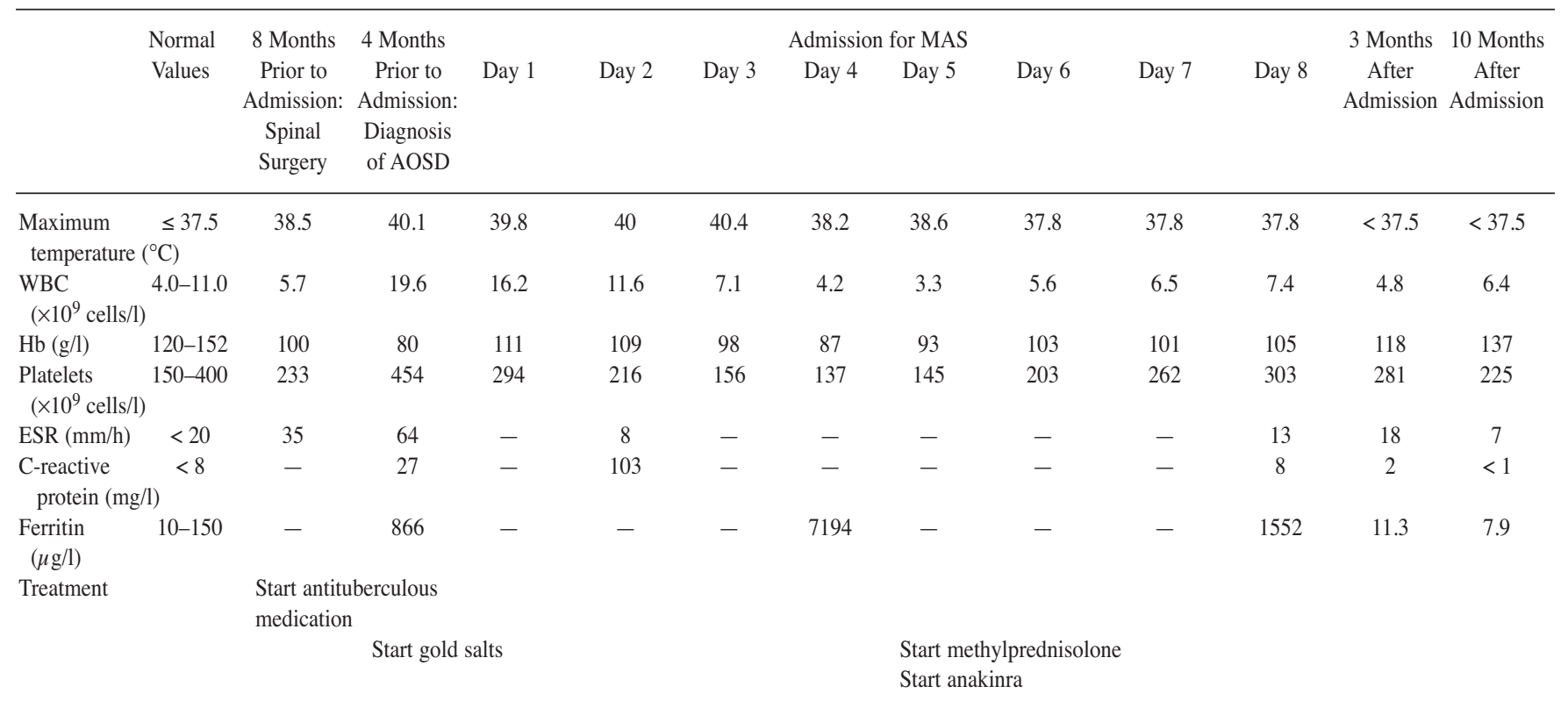

AOSD: adult-onset Still's disease; MAS: macrophage activation syndrome; WBC: white blood cells; Hb: hemoglobin; ESR: erythrocyte sedimentation rate. 


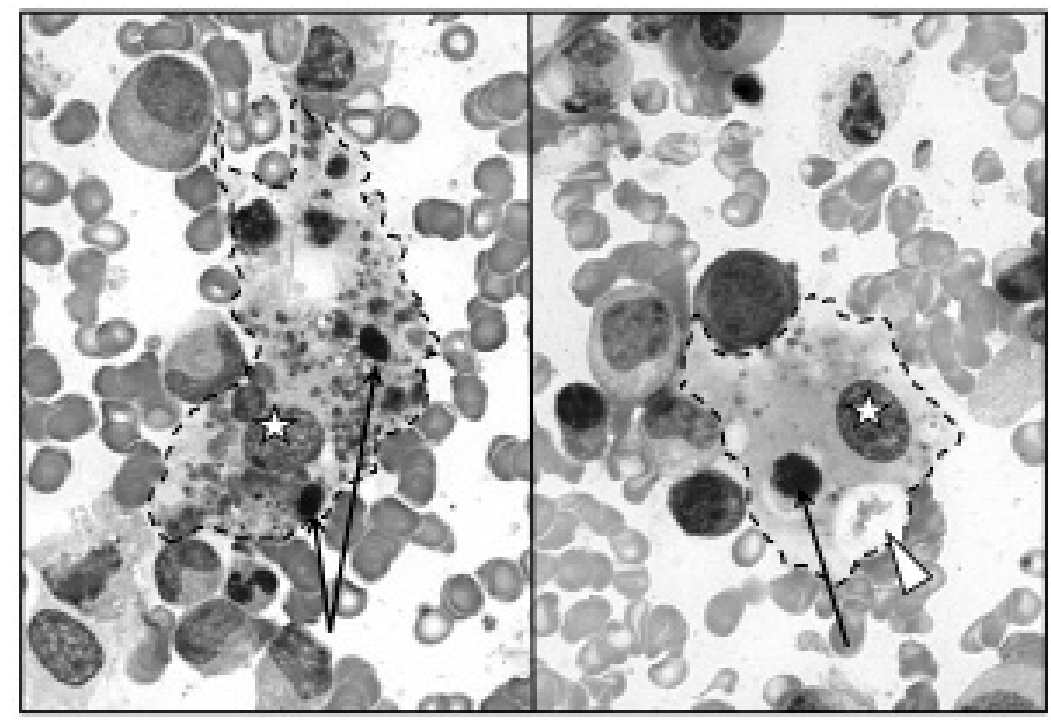

Figure 1. Bone marrow aspirate. Broken lines show cytoplasm of histiocytes. White stars show nuclei of histiocytes. Arrows show intracytoplasmic phagocytized nuclei of hematopoietic precursors. White arrowhead shows ghost of phagocytized hematopoietic precursor.

The use of anakinra to treat refractory AOSD is well established ${ }^{9,10}$. Yet only 1 case report states the efficacy of anakinra as a treatment of MAS, in a 13-year-old patient with systemic-onset juvenile idiopathic arthritis ${ }^{11}$. To our knowledge, this is the first adult case of effective treatment of MAS with anakinra.

This is the first case report of the use of anakinra in a patient with instrumented vertebral fusion for tuberculosis. At 10 months of followup, there has been no recurrence of tuberculosis. We conclude that macrophage activation syndrome in the setting of AOSD can be treated with anakinra.

MADELEINE DURAND, MD, Department of Internal Medicine; YVES TROYANOV, MD, Department of Rheumatology; PIERRE LAFLAMME, MD; GENEVIEVE GREGOIRE, MD, Department of Microbiology, Hôpital du Sacré-Coeur, 5400 Blvd Gouin West, Montreal, Quebec H4J 1C5, Canada.

Address correspondence to Dr. Durand;

E-mail: madeleine.durand@gmail.com

\section{REFERENCES}

1. Wouters JM, van de Putte LB. Adult-onset Still's disease; clinical and laboratory features, treatment and progress of 45 cases. Q J Med 1986;61:1055-65.

2. Fautrel B. Adult-onset Still disease. Best Pract Res Clin Rheumatol 2008;22:773-92.

3. Efthimiou P, Paik PK, Bielory L. Diagnosis and management of adult onset Still's disease. Ann Rheum Dis 2006;65:564-72.

4. Arlet JB, Le TH, Marinho A, Amoura Z, Wechsler B, Papo T, et al. Reactive haemophagocytic syndrome in adult-onset Still's disease: a report of six patients and a review of the literature. Ann Rheum Dis 2006;65:1596-601.

5. Tristano AG. Macrophage activation syndrome: a frequent but under-diagnosed complication associated with rheumatic diseases. Med Sci Monit 2008;14:RA27-36.

6. Ravelli A, Magni-Manzoni S, Pistorio A, Besana C, Foti T, Ruperto $\mathrm{N}$, et al. Preliminary diagnostic guidelines for macrophage activation syndrome complicating systemic juvenile idiopathic arthritis. J Pediatr 2005;146:598-604.

7. Henter JI, Elinder G, Ost A. Diagnostic guidelines for hemophagocytic lymphohistiocytosis. The FHL Study Group of the Histiocyte Society. Semin Oncol 1991;18:29-33.

8. Felix FH, Leal LK, Fontenele JB. Cloak and dagger: the case for adult onset Still disease and hemophagocytic lymphohistiocytosis. Rheumatol Int 2009;29:973-4.

9. Kotter I, Wacker A, Koch S, Henes J, Richter C, Engel A, et al Anakinra in patients with treatment-resistant adult-onset Still's disease: four case reports with serial cytokine measurements and a review of the literature. Semin Arthritis Rheum 2007;37:189-97.

10. Lequerre T, Quartier P, Rosellini D, Alaoui F, De Bandt M, Mejjad $\mathrm{O}$, et al. Interleukin-1 receptor antagonist (anakinra) treatment in patients with systemic-onset juvenile idiopathic arthritis or adult onset Still disease: preliminary experience in France. Ann Rheum Dis 2008;67:302-8.

11. Kelly A, Ramanan AV. A case of macrophage activation syndrome successfully treated with anakinra. Nat Clin Pract Rheumatol 2008;4:615-20.

J Rheumatol 2010;37:4; doi:10.3899/jrheum.091046 